\title{
Quantum phase transitions in cuprates: stripes and antiferromagnetic supersolids
}

\author{
J. Zaanen * \\ Institute Lorentz for Theoretical Physics, Leiden University, P.O. Box 9506, NL-2300 RA Leiden, The Netherlands
}

\begin{abstract}
It is believed that the magnetic fluctuations in cuprate superconductors reflect the proximity to a quantum phase transition. It will be argued that this notion acquires further credibility if combined with the idea that the superconducting state is in a tight competition with the stripe phase over a large range of hole concentrations. On basis of existing data and some simple considerations, a zero-temperature phase diagram will be proposed with an unusual topology which is unique to the competition stripe phase superconductivity. It is argued that the existence of a state which is at the same time stripe ordered and superconducting (antiferromagnetic supersolid) is a prerequisite for quantum critical behavior in the magnetic sector. Various predictions follow which can be tested experimentally. (C) 1999 Elsevier Science B.V. All rights reserved.
\end{abstract}

PACS: 64.60. - i; 71.27.+ a; 74.72. $-\mathrm{h} ; 75.10 .-\mathrm{b}$

Keywords: Quantum phase; Antiferromagnetic; Cuprate superconductors

\section{Introduction}

Until not long ago, it was assumed that cuprate physics was about a rather anomalous metallic state, subjected to a superconducting instability, and a Mott-insulating antiferromagnetic state in a remote corner of the phase diagram. A consequence of the discovery of the stripe phase [1] is that stripes have to be added to the list of states which compete at zero temperature. Although still littered with uncertainties, enough experimental information is available to conjecture the general shape of the zero-temperature $\left(k_{\mathrm{B}} T=0\right)$ phase diagram (see Fig. 1). The $x$ axis has the usual meaning of hole concentration and

\footnotetext{
* Corresponding author. Tel.: + 31-71-527-5506; Fax: + 31-71527-5511; E-mail: jan@1orentz.leidenuniv.nl
}

the other axis is taken in a rough sense as an influence which helps charge localization over superconductivity: I call this $g^{-1}$ since it is similar to the inverse of the coupling constant of a quantum phase-dynamics problem.

As will be further discussed in Section 2, it is about a metal competing with the superconductor at high dopings, about presumably some nickelate-like 'classical' stripes at very low dopings which are strongly affected by quenched disorder and, last but not least, by an 'underdoped regime' where over a large concentration range the superconductor competes with the stripe phase. Although still quite controversial, it might be that at intermediate $g^{-1}$ stripes and superconductivity coexist in this underdoped regime [2]. A main aim of this contribution is to analyze the role of this 'coexistence' or, more precisely, 'antiferromagnetic supersolid' phase. 


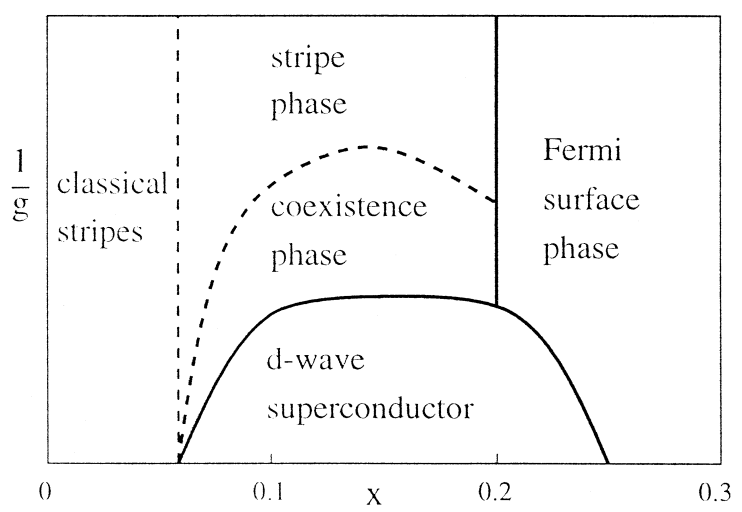

Fig. 1. The topology of the zero-temperature phase diagram of high $T_{\mathrm{c}}$ superconductors, as function of doping $(x)$ and a control parameter $\left(g^{-1}\right)$ suppressing superconductivity and/or promoting the stripe phase (magnetic fields, the LTT deformation, Zn doping).

At stake is that zero temperature ('quantum') phase transitions can govern the physics at finite times, lengths and temperatures, also if one is away from the locus of the transition in $T=0$ parameter space. If the transition is continuous, and if the competition can be described in terms of a bosonic field theory, one meets the phenomena often referred to by quantum criticality $[3,4]$. A generic property of the quantum critical regime is that the phase-relaxation time $\tau_{\phi} \simeq \hbar / k_{\mathrm{B}} T$ while for an effectively Lorentz invariant dynamics the correlation length $\xi$ is related to a geometrical average of energy $\omega$ and temperature: $1 / \xi^{2} \sim(\hbar \omega)^{2}+\left(k_{\mathrm{B}} T\right)^{2} \quad[3,4]$. Using inelastic neutron scattering, Aeppli et al. demonstrated recently that this scaling behavior is obeyed by the incommensurate magnetic fluctuations of $\mathrm{La}_{1.85} \mathrm{Sr}_{0.15} \mathrm{CuO}_{4}$ in its normal state [5]. Since these fluctuations are found at the same wavenumbers as the magnetic superlattice Bragg peaks of the static stripe phase [2,6], it is tempting to think that these fluctuations have to do with the proximity of the stripe antiferromagnetic order. This interpretation is further helped by the observation that the spectrum of incommensurate fluctuations acquires a gap at low temperatures, and this gap $\Delta \varepsilon$ is very small $(6 \mathrm{meV})$ as compared to the lattice scale exchange $(100 \mathrm{meV})$ [7]: the smallness of this gap signals the close proximity to the quantum critical point. In addition, it has been argued that the antiferromagnet found in the
LTT cuprates $\mathrm{La}_{2-x-y} \mathrm{RE}_{y} \mathrm{Sr}_{x} \mathrm{CuO}_{4} \quad(\mathrm{RE}=\mathrm{Eu}$, $\mathrm{Nd}, \ldots)$ is characterized by strong quantum fluctuations [8], indicating the proximity of the stripe antiferromagnet itself to the quantum disordering transition.

The above interpretation points at the presence of a second order quantum phase transition, at least involving the spin sector. As I will show, this observation together with the phase diagram of Fig. 1 puts some strong constraints on the form of the effective low energy theory. The argument rests on: (a) Some well established notions developed in the context of the strongly interacting boson problem, centered around the concept of supersolid order [9-13]. (b) a straightforward extension to the $T=0$ (quantum) case of the phenomenological theory by Zachar et al. [14] for stripe order. These matters will be discussed in Section 3. Since the phase diagram Fig. 1 has, to my knowledge, not been proposed before, let me first discuss its somewhat uncertain status.

\section{Topology of the zero-temperature phase dia- gram}

The assumption underlying the construction of Fig. 1 is that the 'perturbations' stabilizing stripe order can all be understood as the ' $g^{-1}$ ' ( $y$-axis) of Fig. 1. This is not quite obvious, and even if true, the physically realizable $g^{-1}$ parameters are not well behaved, with the effect that big portions of the phase diagram have not yet been accessed. The best documented ' $g^{-1}$ ' is the rare earth concentration $y$ in the cuprates of composition $\mathrm{La}_{2-x-y} \mathrm{RE}_{y} \mathrm{Sr}_{x} \mathrm{CuO}_{4}$ $(\mathrm{RE}=\mathrm{Nd}, \mathrm{Eu}$, etc.) showing the low temperature tetragonal (LTT) distortion. As argued by Tranquada et al. [1], the LTT deformation can be regarded as a relatively weak collective pinning potential. If this potential could be switched on continuously, it would be close to an ideal realization of $g^{-1}$. The problem is, however, that at a critical substitution $y_{\mathrm{c}}$ the LTT deformation switches on in a first order transition $[15,16]$ as expected for a $3 \mathrm{D}$ structural transition. Apparently, this corresponds to a jump from deep inside the superconducting regime into the coexistence regime of Fig. 1. A next candidate is substitution by impurities like $\mathrm{Zn}$ [17]. The problem is that 
this introduces additional quenched disorder into the problem, further obscuring the clean limit physics $[18,19]$. Finally, magnetic fields [20] are believed to stabilize stripes also. Besides the practical problem that few experiments can be done in $\sim 60 \mathrm{~T}$ fields, additional complexities are expected here also. ${ }^{1}$ It is a matter of high priority for the experimental community to search for alternative $g^{-1}$ like control parameters.

Given these reservations, the phase diagram topology follows directly from experiments. The metal-insulator transition at $x \simeq 0.20$, as seen in magnetic fields [20] (and $\mathrm{Zn}$ substitution experiments [22] coincides with the concentration where Tranquada et al. [2] find the stripe order parameter to disappear in the LTT system. It is firmly established that in the concentration range $x=0.125-0.20$ incommensurate magnetic order is present in the LTT system [2] and some evidence is available for the presence of this order at $x<1 / 8$, even in $\mathrm{La}_{2-x} \mathrm{Sr}_{x} \mathrm{CuO}_{4}[23,24]$ itself. A second singular doping concentration is $x \simeq 0.06$ where the superconductivity disappears. Remarkably, Yamada et al. [6] find that with the diminishing of the superconductivity also the incommensurate magnetic fluctuations disappear, being replaced by a broad peak centered at the $(\pi / a, \pi / a)$ wavevector. Although evidence exists showing that one or the other collective phenomenon involving the holes and the spins is at work in the doping range $0<x<0.06$ [25,26], it remains to be seen if this is related to the stripes at higher doping. Finally, a crucial issue is whether the superconductor and the stripe phase are separated by an intervening microscopic coexistence phase (see Fig. 1). The experimental situation [2] is far from settled, and a main purpose of this communication is to discuss the possible role of this coexistence phase. However, assuming that it exists, it is clear that for increasing ' $g$ ' , the superconductivity will eventually vanish. It has been shown that for increasing

\footnotetext{
${ }^{1}$ Given Fig. 1, it is expected that the cores of the superconducting vortices are antiferromagnetic (see Ref. [21]). The magnetic field driven superconductivity-stripe transition could be related to a percolation-like transition where the vortex cores start to overlap.
}

LTT tilt angle in the $\mathrm{La}_{2-x-y} \mathrm{Sr}_{x} \mathrm{Nd}_{y} \mathrm{CuO}_{4}$ system a region opens up around $x=1 / 8$ which is not superconducting $[15,16]$.

The novelty of the phase diagram (Fig. 1) is that as function of doping lines of $T=0$ phase transitions are present, instead of the isolated points which are discussed in the theoretical literature. It is experimental fact that stripe phases exists in a large doping range [2]. Different from Mott-Hubbard insulators, the charge- and spin order exists away from points of low order charge commensuration. Although the ordering seems characterized by a partial commensuration $[27,28]$, what matters in the first instance is that stripes can be formed in a large range of dopings. Because the superconducting order is not critically dependent on the hole density either, quantum phase transitions can occur over a wide range of dopings. This helps to remove a standard difficulty associated with the idea that high- $T_{\mathrm{c}}$ superconductivity is related to the physics of quantum phase transitions. The quantum criticality as referred to in Section 1 is apparently present over a large doping range, and this is not natural if the physics is controlled by an isolated quantum critical point on the doping axis. However, it becomes more natural given that there is a line of critical points as function of doping.

Ignoring the low doping regime, in addition to the line of stripe related transitions there is a single isolated singular point as function of doping: the metal-insulator transition at $x \simeq 0.20$ [49]. The $T=0$ phase diagram of Fig. 1 actually suggests a particular interpretation of the finite temperature crossover diagram as constructed by Pines et al. [29], based on the analysis of a vast amount of data. This cross-over diagram is reproduced in Fig. 2. The spin-gap temperature $T^{*}$ (dashed line) can be interpreted as measuring the 'distance' between the superconductor and the coexistence phase. If temperature exceeds the spin gap the ' $z=1$ ' quantum critical regime is entered, which is associated with the freezing of the stripe antiferromagnetism. It is obvious that this spin gap will, at least initially, grow as function of increasing doping. However, there are also crossover lines associated with the singular $x=x_{\mathrm{MI}}$ of the metal insulator transition: $T_{\text {cr }}$ (full lines) [30,31]. In sharp contrast with $T^{*}, T_{\text {cr }}$ is strongly doping dependent, as expected for a crossover line associated with an isolated point on the doping axis [50]. 


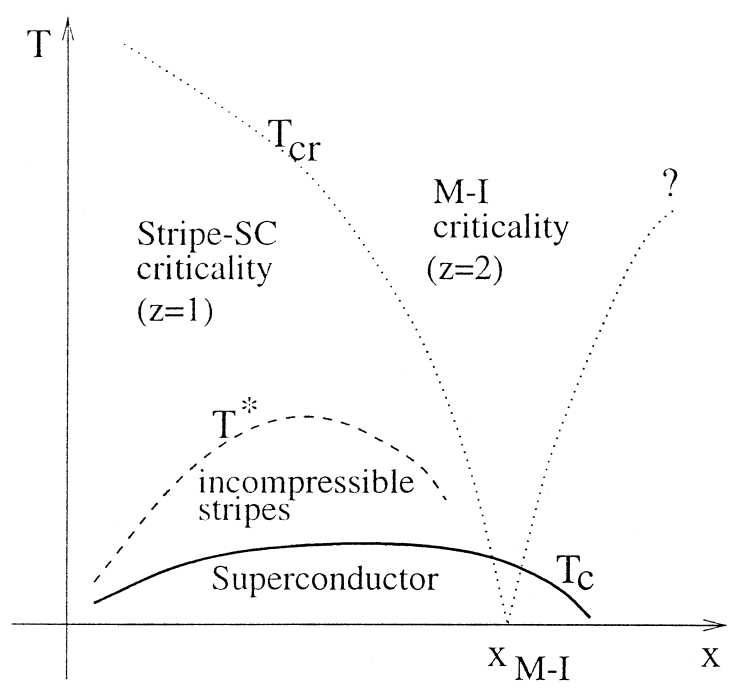

Fig. 2. Finite temperature crossover diagram according to Pines [29], but now with an interpretation motivated by the zero temperature phase diagram (Fig. 1). The dotted lines $\left(T_{\mathrm{cr}}\right)$ indicate the crossover to the quantum critical regime controlled by the metalinsulator transition. To the underdoped side, a regime is entered below $T_{\text {cr }}$ which is controlled by the line of quantum phase transitions from the superconductor to the coexistence phase. The spin-gap temperature $T^{*}$ measures the $T=0$ 'distance' to the coexistence phase.

The precise nature of the critical regime associated with the metal-insulator transition depends on the nature of the metal in the overdoped regime. Assuming that this metal is a Fermi-liquid, the critical regime is likely of the Millis-Hertz variety $[32,33]$ as controlled by the vanishing of the stripe order. Such an interpretation acquires further credibility by the observation that this regime is characterized by mean field exponents $(z=2)$, and the remaining issue is if the transition is dominated by the spinchannel [29] or the charge channel [34]. Obviously, it remains to be seen if high $T_{\mathrm{c}}$ superconductivity has anything to do with Fermi-liquid physics [35].

\section{Criticality and the antiferromagnetic supersolid}

Let us now focus on the doping regime characterized by the competition between superconductivity and the stripe phase. It is assumed that the long wavelength dynamics is governed by conventional bosonic ordering fields, described in terms of a
Ginzburg-Landau-Wilson (GLW) action. A next assumption is that the stripe-antiferromagnet orders in a continuous quantum phase transition. This is motivated by the work of Aeppli et al. [5] as discussed in Section 1. Leaning heavily on the well understood phenomenology of supersolid order, together with the work by Zachar et al. [14] on the phenomenology of stripe ordering, I find that the demand for a continuous transition acts as a strong constraint on the allowed dynamics. First order behavior is more natural in the present context, and only under quite specific circumstances second order transitions can occur. The analysis which follows is not complete. At several instances a full renormalization group (RNG) analysis is still to be done, but it is not expected that this will change the picture radically. Quenched disorder is neglected altogether. For a two-dimensional order like the stripe phase, quenched disorder has to dominate eventually [36]. However, because the disordering lengths associated with the static stripe phases tend to be rather large, it should make sense to analyze first the clean limit, while disorder physics becomes only of relevance very close to the phase transition. This section is organized as follows: first I will introduce a minimal set of ordering fields (Section 3.1). In the absence of the spin fields, the problem becomes quite similar to the problem of supersolids, which will be discussed next (B). The charge-spin coupling will be discussed, following the work of Zachar et al. (C), and combined with the supersolid theme in Section 3.4.

\subsection{The ordering fields}

On the level of GLW-theory, the phase diagram Fig. 1 suggests a rather rich dynamics because of the involvement of a variety of ordering fields. The order parameters of relevance are as follows.

(i) The spatially uniform d-wave superconducting order parameter $\left\langle\mathrm{e}^{i \theta_{0}}\right\rangle$, parametrized in terms of the phase-angle $\theta_{0}$. The phase angle $\theta_{0}$ is conjugate to the uniform charge density $N_{0}$ such that $\left[N_{0}, \theta_{0}\right]=i$.

(ii) The finite wavevector charge density wave order $\vec{N}_{2 \varepsilon}$ associated with the stripe phase charge order. The total charge density can be written as,

$$
N(x)=N_{0}+N_{2 \varepsilon, 1} \cos \left(2 \varepsilon x_{1}\right)+N_{2 \varepsilon, 2} \cos \left(2 \varepsilon x_{2}\right)
$$


$2 \varepsilon$ is the wavevector of the charge order, while the stripe phase can occur in two orientations $\left(x_{1,2}\right.$ are the $(1,0)$ and $(0,1)$ directions in the lattice, respectively). The implication is that $N_{2 \varepsilon}$ is a vector: $\vec{N}_{2 \varepsilon}=\left(N_{2 \varepsilon, 1}, N_{2 \varepsilon, 2}\right)$. As under (i), superfluid phase angles $\theta_{2 \varepsilon, i}(i=1,2)$ are conjugated with the charge order, corresponding with finite momentum superconductivity: $\left[N_{2 \varepsilon, i}, \theta_{2 \varepsilon, j}\right]=i \delta_{i j}$. The interplay of charge density wave order and superconductivity is the central theme in the literature dealing with supersolid order.

(iii) The novelty is the incommensurate antiferromagnetic spin order associated with the stripe phase. A crucial issue is if the spin order is collinear, with the spatial modulation of the staggered order parameter driven by the magnitude of the staggered magnetization, or if some spiral modulation is involved. For the collinear case, the relevant long wavelength theory is the same as, e.g., a simple two sublattice Heisenberg antiferromagnet $(O(3)$ quantum non-linear sigma model, or the 'soft spin' model adapted here) while the fluctuations of spiral phases are described by more involved matrix models [37]. Although direct experimental evidence is not available, it is generally believed that the stripe-antiferromagnet in the cuprates is of the collinear variety, both because this is the unanimous outcome of theoretical work [38], and because of the experience in the nickelates [39]. The staggered spin density is,

$\vec{M}(x)=\vec{M}_{\varepsilon, 1} \cos \left(\varepsilon x_{1}\right)+\vec{M}_{\varepsilon, 2} \cos \left(\varepsilon x_{2}\right)$

defining the $O(6)$ rotor field $\vec{M}_{\varepsilon}=\left(\vec{M}_{\varepsilon, 1}, \vec{M}_{\varepsilon, 2}\right)$ where $\varepsilon$ refers to the modulation wavevector, $i=1,2$ to the stripe orientation, and $\vec{M}_{\varepsilon, i}=\left(M_{\varepsilon, i}^{x}, M_{\varepsilon, i}^{y}, M_{\varepsilon, i}^{z}\right)$

\subsection{Phenomenology of the supersolid}

In the absence of spin-order, the remaining charge sector is similar to the well studied subject of supersolid order. Although the microscopic physics behind the stripe phenomenon is clearly quite different from the simple Bose-Hubbard models discussed in the latter context, there is no obvious reason to expect the long-wavelength behavior to be different. In the absence of antiferromagnetism, the progression superconductor-coexistence phase-stripe phase of Fig. 1 translates in the triad superconductor-supersolid- collinear charge order known from the study of Bose-Hubbard models [9-11,16]. Let me recollect some results as of relevance to the present context.

The starting point is the Bose-Hubbard model,

$$
\begin{aligned}
H= & J \sum_{\langle i j\rangle}\left(a_{i}^{\dagger} a_{j}+a_{j}^{\dagger} a_{i}\right)-\mu \sum_{i} n_{i}+U_{0} \sum_{i} n_{i}^{2} \\
& +U_{1} \sum_{\langle i j\rangle} n_{i} n_{j}+U_{2} \sum_{\langle i k\rangle} n_{i} n_{k}
\end{aligned}
$$

where $a_{i}^{\dagger}$ and $a_{i}$ are bosonic creation and annihilation operators obeying $\left[a_{i}, a_{j}^{\dagger}\right]=\delta_{i j}\left(n_{i}=a_{i}^{\dagger} a_{i}\right)$. The parameters $t, \mu$ and $U_{0}$ are the hopping, thermodynamic potential and on-site interaction, respectively, while $U_{1}$ and $U_{2}$ are nearest-neighbor and nextnearest-neighbor interactions. This model has a literal interpretation in the context of Josephson junction networks, while in the present context it is no more than a convenient lattice cut-off model, revealing universal features of the long wavelength physics.

In the absence of the non-local interactions $U_{1,2}$ this model describes the competition between the condensation of the $q=0$ charge mode (Mott-insulator) and the superfluid. For $U_{1,2} \neq 0$ charge density wave order is found at particular densities. If $U_{2} \geq U_{1}$ a particular charge ordering occurs which is of interest in the present context: a stripe charge order becomes stable (often called 'collinear' in the BoseHubbard literature). In Fig. 3 a representative part of the phase diagram is sketched [12], as function of increasing kinetic energy $\left(J / U_{0}\right)$ and average particle number $\left(n_{0}\right)$ in the grand canonical ensemble, for some particular choice of non-local interactions. At integer fillings $\left(n_{0}=0,1, \cdots\right)$ the uniform Mottinsulating (MI) state is stable for small kinetic energy, while the stripe state ( $\mathrm{Sol}$ ) acquires stability at half-integer fillings $\left(n_{0}=1 / 2,3 / 2, \cdots\right)$. Upon increasing the kinetic energy, first a phase is entered characterized by a coexistence of stripe order and superfluidity: the stripe (or collinear) supersolid (Ssol). Upon a further increase of $J$ the stripe order weakens to disappear at the phase boundary with the pure superfluid (SF). It is noticed that the BoseHubbard collinear order has much in common with the cuprate stripe order. For instance, the bondordered stripes as found by White and Scalapino [40] in their numerical studies of the $t-J$ model (Fig. 4) are quite like the Bose-Hubbard collinear states 


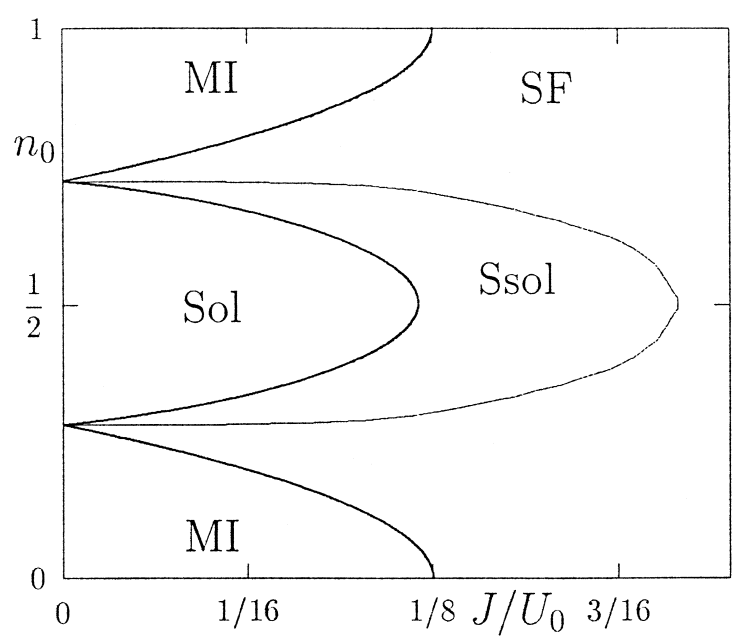

Fig. 3. The mean-field phase diagram of the Bose-Hubbard model as function of $J / U_{0}$ and average boson density $n_{0}$, according to van Otterlo et al. [12]. Numerical studies indicate that the topology of the phase diagram does not change significantly due to the fluctuations in $2+1 \mathrm{D}$, for both the checkerboard [12] and collinear/stripe [9] charge orders.

assuming that the electrons pair on the elementary plaquet to form effective bosons. ${ }^{2}$ Interestingly, a simple explanation is found within this framework for the doping independence of the stripe wavevector $\varepsilon$ in the doping regime $1 / 8<x<0.20$ [2]. The stripe phase of the Bose-Hubbard model occurs in the classical limit $(t=0)$ only at a half-integer filling, with the associated commensurate wavevector $\pi / 2 a$ ( $a$ is the lattice constant). The system would phase separate at non- (half) integer fillings in Mottinsulating and stripe regions. However, the supersolid phase can exist in a homogeneous form away from half integer filling, keeping the wavevector of the charge order commensurate with the underlying lattice: also away from charge commensuration the density wave can stay commensurate because the excess particle density can be 'eaten' by the superfluid order. Notice that the optimal stability of the stripe phase of Fig. 3 occurs at half-integer filling;

\footnotetext{
${ }^{2}$ It has been argued that the 'half-filled' cuprate stripes might develop an on-stripe density wave instability [41-43]. Pending microscopic details, these could give rise in principle to secondary transitions in the charge sector.
}

this is quite like the special stability of the cuprate stripes at the commensurate density $x=1 / 8$.

A subtle issue is the role played by the finite momentum superconductor, $\left\langle\mathrm{e}^{i \theta_{2 \varepsilon}}\right\rangle$. In the BoseHubbard context, this is playing no role. In fact, by letting the superconductivity live at $q=0$ and the charge-order at finite wavevector the either-or competition is avoided which is a consequence of the number operator being conjugate to the phase, and this makes possible the existence of the supersolid. Self-evidently, since translation symmetry is broken by the charge order, the superconducting order also acquires a spatial modulation commensurate with the charge order. However, this involves the amplitude of the SC order parameter which acquires an admixture with a finite momentum component. However, this component is parasitic and does not play a critical role. I will assume that this is also the case in the cuprates.

Let us now discuss the nature of the phase transitions of Fig. 3. Obviously, in the absence of the intervening supersolid phase, the transition between the stripe phase and the superconductor would be first order. The intervention of the supersolid, on the other hand, allows in principle for the occurrence of continuous quantum phase transitions. Although sec-

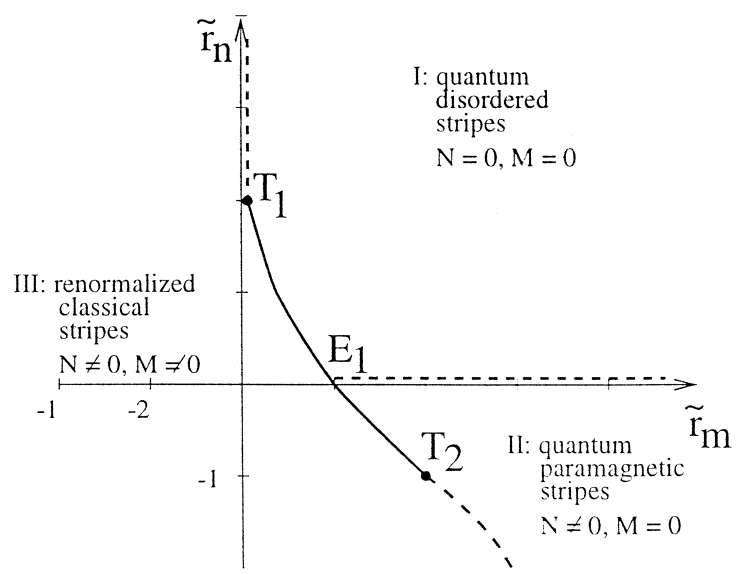

Fig. 4. The mean-field phase diagram following from the stripe action Eqs. (4), (8) and (9) according to Zachar et al. [14], here interpreted as a zero-temperature phase diagram. The axis are the coupling constants of the charge $\left(\tilde{r}_{n}=r_{N} / \lambda_{1}^{2}\right)$ and $\operatorname{spin}\left(\tilde{r}_{m}=\right.$ $\left.r_{M} / \lambda_{1}^{2}\right)$ sectors, respectively. Dashed lines refer to second order transitions and the heavy line corresponds with the spin-charge coupling induced first order transitions. 
ond order transitions are found on the mean field level, Frey and Balents [13] presented an interesting analysis showing that the role of critical fluctuations is subtle. For future use, let me review their arguments. The Ginzburg-Landau-Wilson (GLW) action consistent with the symmetries of $\vec{N}_{2 \varepsilon}=$ $\left(N_{2 \varepsilon, 1}, N_{2 \varepsilon, 2}\right)$ (Eq. (1)) is

$$
\begin{aligned}
S_{N}= & \int \mathrm{d} x \mathrm{~d} \tau\left\{\frac { 1 } { 2 } \sum _ { i = 1 } ^ { 2 } \left[\left(\frac{1}{c_{N}} \partial_{\tau} N_{2 \varepsilon, i}\right)^{2}+\left(\nabla N_{2 \varepsilon, i}\right)^{2}\right.\right. \\
& \left.+r_{N} N_{2 \varepsilon, i}^{2}\right]+\frac{u_{N}}{4 !}\left(\sum_{i} n_{2 \varepsilon, i}^{2}\right)^{2} \\
& \left.-\frac{w_{N}}{4 !}\left(\sum_{i} N_{2 \varepsilon, i}^{4}\right)\right\}
\end{aligned}
$$

where $c_{N}$ is a velocity characterizing the charge order, while the mass $r_{N}$ measures the distance from the critical point associated with the charge ordering. For the quartic anisotropy parameter $w_{N}=0$ this would correspond (at $k_{\mathrm{B}} T=0$ and 2 space dimensions) with the GLW action of a classical $X Y$ system in $D=3$. If the anisotropy $w_{N}>0$, stripes oriented along $(1,0)$ or $(0,1)$ are favored in the ordered state.

The superfluid order parameter corresponds with $\left\langle\mathrm{e}^{i \theta_{0}}\right\rangle$, where the superfluid phase $\theta_{0}$ is governed by the usual quantum phase dynamics,

$S_{S}=\frac{1}{2 g_{S}} \int \mathrm{d} x \mathrm{~d} t\left[\left(\frac{1}{c_{S}} \partial_{\tau} \theta_{0}\right)^{2}+\left(\nabla \theta_{0}\right)^{2}\right]$,

at least deep in the superconducting phase. It is noticed that the transition between the supersolid and the stripe phase is actually governed by a dilute Bose-gas action [44] away from points of charge commensuration $[3,4,12]$. The physical interpretation is that mobile bosonic defects in the stripes deconfine and these form initially a dilute gas of bosons. Since the stripe phase excitation spectrum is characterized by a commensuration gap, the stripe phase order parameter acts like a spectator at this transition.

Of more interest is the transition between the supersolid and the superconductor. Because of the massless character of the phase fluctuations, these can in principle interfere with the critical fluctuations associated with the charge-ordering transition. The lowest order allowed coupling between the phase and the charge order parameter is,

$S_{N S}=\int \mathrm{d} x \mathrm{~d} \tau i \sigma_{N}\left(\partial_{\tau} \theta_{0}\right) \sum_{i} N_{2 \varepsilon, i}^{2}$

Frey and Balents [13] show that the critical fluctuations renormalize the phase velocity $c_{S}$ (Eq. (5)) according to,

$c_{S, R}^{2}=\frac{c_{S}^{2}}{1+\text { const. } \xi^{D \alpha /(2-\alpha)}}$

where $D=3$ (space-time dimensionality) and $\xi$ the correlation length associated with the stripe ordering. $\alpha$ is the specific heat exponent and it is seen from Eq. (7) that for $\alpha>0 \quad c_{S, R}^{2} \rightarrow 0$ at the transition, signalling a runaway flow, while for $\alpha<0$ the coupling Eq. (6) is irrelevant. It is a classic result of renormalization group theory [45] that the quartic anisotropy $w_{N}$ in Eq. (4) is irrelevant at this transition. The transition falls therefore in the $D=3 X Y$ universality class, and since the specific heat exponent is negative, the coupling to the superfluid phase mode is irrelevant also.

Summarizing, although the direct transition from the superconductor to the stripe phase is first order, in the presence of a supersolid two continuous quantum phase-transitions are found: the superconductor-supersolid transition is a 3D XY transition, and the supersolid-stripe transition is generically described by the dilute bose gas.

\subsection{Phenomenology of quantum stripes}

As compared to Section 3.2, the novelty of the cuprate stripe phase is the prominent role of antiferromagnetism. Neglecting superconductivity, the problem remains of the interplay of the finite wavevector charge- and spin modes and this has been analyzed on the phenomenological level by Zachar et al. [14]. This work focuses on the finite temperature classical phase diagram, but it is easily generalized to the $2+1 \mathrm{D} k_{\mathrm{B}} T=0$ quantum dynamics.

The zero-temperature dynamics of the stripe-antiferromagnetic order parameter $\vec{M}_{\varepsilon}$ (Eq. (2)) can be 
represented by a 'soft-spin' GLW action, which is the six-flavor version of the charge action, Eq. (4),

$$
\begin{aligned}
S_{M}= & \int \mathrm{d} x \mathrm{~d} \tau\left\{\frac { 1 } { 2 } \sum _ { i = 1 } ^ { 6 } \left[\left(\frac{1}{c_{M}} \partial_{\tau} M_{\varepsilon, i}\right)^{2}+\left(\nabla M_{\varepsilon, i}\right)^{2}\right.\right. \\
& \left.+r_{M} M_{\varepsilon, i}^{2}\right]+\frac{u_{M}}{4 !}\left(\sum_{i} M_{\varepsilon, i}^{2}\right)^{2} \\
& \left.-\frac{w_{M}}{4 !}\left(\vec{M}_{\varepsilon, 1} \cdot \vec{M}_{\varepsilon, 1}+\vec{M}_{\varepsilon, 2} \cdot \vec{M}_{\varepsilon, 2}\right)^{2}\right\} .
\end{aligned}
$$

The quartic anisotropy $w_{M}$ is chosen such that it leaves the internal $O(3)$ spin rotation unaffected, breaking the spatial rotation symmetry to $Z_{2}$; overall, $O(6)$ is broken by $w_{M}$ to $O(3) \times Z_{2}$. As shown by Brezin et al. [45], any quartic anisotropy is relevant at the phase transition of a $O(N)$ problem with $N>4$. Since $N=6$ for the action Eq. (8), its phase transition is governed by $O(3) \times Z_{2}$ universality. Little attention has been paid to such symmetry breakings in the statistical physics literature and the precise nature of its quantum critical regime is under investigation.

The actions Eqs. (4) and (8) describe the ordering of the stripe charge- and spin fields independently. Because a fully developed stripe phase is at the same time charge- and spin ordered, the mode couplings between these fields should be included. These have been analyzed by Zachar et al. [14]. Their findings can be directly applied to the present context of quantum phase transitions. Including the twofold degeneracy related to the stripe orientation, the lowest order allowed spin-charge mode couplings are,

$$
\begin{aligned}
S_{N M}= & \int \mathrm{d} x \mathrm{~d} \tau\left\{\frac{\lambda_{1}}{2} \sum_{i=1}^{2}\left[N_{2 \varepsilon, i}^{*} \vec{M}_{\varepsilon, i} \vec{M}_{\varepsilon, i}+\text { h.c. }\right]\right. \\
& \left.+\frac{\lambda_{2}}{2} \sum_{i=1}^{2}\left|N_{2 \varepsilon, i}\right|^{2}\left|\vec{M}_{\varepsilon, i}\right|^{2}\right\}
\end{aligned}
$$

The leading order spin-charge coupling $\lambda_{1}$ is proportional to the charge field itself and to the square of the spin-field, because the former is a scalar and the latter is a vector. This explains directly why spin orders at the wavevector $\varepsilon$ and the charge at $2 \varepsilon$. The coupling Eq. (9), together with Eqs. (4) and (8), defines a phenomenological theory for stripe ordering,

$S_{\text {stripes }}=S_{N}+S_{M}+S_{N M}$.

On the mean-field level, the coupling $\lambda_{1}$ gives rise to a rich phase diagram, which is reproduced in Fig. 4. Although still to be confirmed by a full RNG analysis, it is expected that the topology of this phase diagram will not change in three dimensions if fluctuations are included. This is quite different in two dimensions. Assuming $2 \varepsilon$ to be commensurate with the lattice, and neglecting the orientational freedom, the charge sector is Ising-like and can therefore order at finite temperature. However, the spin sector carries a continuous internal symmetry such that magnetic order is forbidden at any finite temperature according to the Mermin-Wagner theorem. The interpretation by Zachar et al. of the finite temperature phase transitions of LTT cuprates in terms of the phase diagram Fig. 4 was criticized by van Duin and myself [8]. We argued that the stripe antiferromagnet is relatively close to the zero-temperature order-disorder transition, with the effect that the 2D-3D crossover in the magnetic sector is pushed to low temperatures, such that mean-field theory loses its validity.

The quantum ordering dynamics at zero temperature is governed by the (three) dimensionality of space-time and the topology of the mean-field phase diagram (Fig. 4) is not expected to be affected by fluctuations in a significant way. It is therefore expected that a quantum stripe system has the following phases: (a) Phase I $\left(r_{M}, r_{N}>\lambda_{1}^{2}\right)$ : the 'quantum incompressible stripe phase'. Both the spin- and charge sector are quantum disordered. Because the correlation length in the imaginary time direction is finite in both sectors, both the charge- and spin excitation spectrum should show gaps at the stripe wavevectors. This is the interpretation found in the present framework for the 'dynamical stripes' conjectured to exist in cuprate superconductors. (b) Phase II $\left(r_{M} / \lambda_{1}^{2}<1\right.$ and /or $\left.r_{N} / \lambda_{1}^{2}<2\right)$ : the 'renormalized classical stripe phase'. Both spin- and charge are ordered, and this phase corresponds with the 'static' stripe phase. (c) Phase (III) $\left(r_{N}<0\right.$ and $r_{M} / \lambda_{1}^{2}$ larger than a critical value): the 'quantum paramagnetic stripe phase'. Although the charge is ordered, the spin system remains in a quantum disordered 
state, and is characterized by a dynamical mass gap. It is noticed that in principle also a state can exist which is spin ordered and charge disordered but this involves necessarily transversal modulations of the spin system (the circular spiral state of Zachar et al. [14]).

The phase transitions behave in an interesting way as function of the various coupling constants. Starting at $r_{N} \gg \lambda_{1}^{2}$, there is a second order transition between the fully disordered state and the static stripe phase. This transition is driven by the sign change of $r_{M}$ : the spin driven transition. The charge mode is massive $\left(r_{N} \gg 0\right)$ and is unimportant in the critical regime, as will be further discussed in Section 3.4 .

Upon decreasing $r_{N}$, a regime is entered where the thermodynamics becomes driven by the spincharge coupling, Eq. (9), and this causes $\{\backslash$ em first order\} behavior (heavy line in Fig. 4). Initially, this first order transition separates the disordered from the fully ordered stripe phase, but when $r_{N}$ changes sign a second order charge transition splits off ( $E_{1}$ in Fig. 4). For $r_{M}>\lambda_{1}^{2}$ one finds therefore the sequence: quantum disordered stripes, quantum paramagnetic stripe phase, and renormalized classical stripe phase. Initially the spin ordering transition remains first order (due to the mode coupling) to change to a continuous transition in the purely charge driven regime. It is noticed that this latter transition is in the 3D $O(3)$ universality class because the orientational freedom is already broken at the charge transition.

\subsection{Stripes and superconductivity: antiferromag- netic supersolids}

In direct analogy with the coupling between the charge-density mode and the superfluid phase, Eq. (6), the coupling between the uniform superconductor and the stripe-antiferromagnet becomes,

$S_{M S}=\int \mathrm{d} x \mathrm{~d} \tau i \sigma_{M}\left(\partial_{\tau} \theta_{0}\right) \sum_{i=1}^{6} M_{\varepsilon, i}^{2}$

The crucial observation is that the interplay between finite wavevector charge order and zero-momentum superconductivity, as discussed in Section 3.2, can be 'dressed up' with the stripe antiferromagnetism, without changing the picture drastically. On the phenomenological level, the magnetic order parameter can be substituted anywhere for the charge order parameter, with the only difference that the symmetry is becoming larger. In analogy with the supersolid, a pure antiferromagnet and a pure superconductor are separated by a first order boundary. However, a coexistence (antiferromagnetic superconductor) phase is thermodynamically allowed and both the antiferromagnet-coexistence phase and the coexistence phase-superconductor transitions are of second order. In the context of stripes we meet in addition the charge-spin mode couplings causing the rich phase diagram (Fig. 4). Since the charge and spin modes couple in a similar way to the superconductivity, the supersolid (Fig. 3) and stripe (Fig. 4) phase diagrams 'commute' with each other.

First order boundaries are rather natural in the present context and I leave it to the reader to enumerate all possible transitions of this kind. From now on, I insist on the continuous character of the transition involving the ordering of the stripe antiferromagnet, as motivated by the observations in Sections 1 and 2. A first condition is that a coexistence phase should be present; a direct transition from the singlet superconductor to a pure stripe phase is necessarily of first order. The second condition follows from the stripe phase diagram (Fig. 4): the charge-spin driven first-order transitions should be avoided. By these simple considerations I find two possible scenario's which allow for a second order spin ordering transition (Fig. 5).

\subsubsection{Scenario I: Independent transitions}

The trivial way to arrive at a continuous magnetic transition is obviously to let all orderings occur independently. This is possible if the stripe sector is in the 'charge driven' (large $\tilde{r}_{m}$ ) region of the phase diagram (Fig. 4). A typical sequence of quantum phase transitions as function of decreasing ' $g^{-1}$, could be as indicated in Fig. 5a: the superconductor acquires a charge order in the $O(2)$ transition of Frey and Balents [13]. The spin system of this stripe phase is still quantum disordered, and orders independently in a standard $O(3)$ transition. The dilute boson transition where the superconductivity vanishes might happen before or after this spin freezing transition; 
(a)

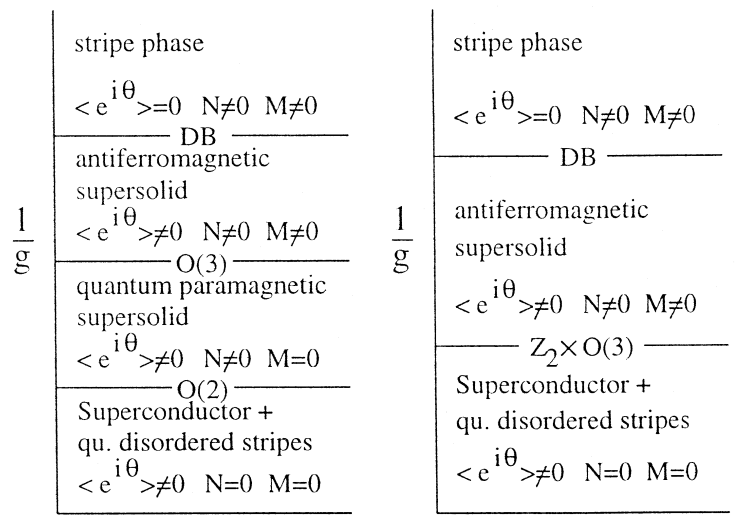

Fig. 5. The two possible scenarios, implied by the presence of a continuous spin ordering transition. The symmetries governing the various phase transitions are also indicated (DB is dilute bosons).

the charge- and spin sectors are in principle governed by independent coupling constants and the order in which the transitions happen is determined by the microscopy. Assuming that the antiferromagnetic supersolid exists, the sequence of the transitions is as indicated in Fig. 5a. This fingerprint of this scenario is a stripe phase which is charge ordered while the spin sector is still quantum disordered: the quantum paramagnetic supersolid, or in other words, a superconducting stripe phase with a spin gap. Although such a state has not been seen in experiments, it has been (implicitly) discussed theoretically by Tworzydlo et al. [46]. The scenario Fig. 5a might appear as less natural for the cuprates. It would be expected that the (quantum) critical fluctuations in the superconducting state would be dominated by the charge dynamics associated with the superconductor-paramagnetic stripe phase transition, and not by the spin fluctuations. At the same time, very little is known experimentally on how the stripe related charge fluctuations behave and this possibility cannot be excluded on basis of the available data.

\subsubsection{Scenario II: The spin-driven stripe ordering}

There is yet another possibility: the spin-driven regime of the stripe phase diagram (Fig. 4). The phase diagram simplifies in this case (Fig. 5b), and becomes literally like the empirical phase diagram shown in Fig. 1. Different from the charge driven case (scenario I), the transition is now from the superconductor directly into the antiferromagnet supersolid. In addition, since the transition is dominantly spin driven this possibility appears as more natural, given the quantum critical spin dynamics observed by Aeppli et al.

Although one would expect the transition from a superconductor to an antiferromagnetic supersolid to be of first order, the transition can be of second order because the coupling term, Eq. (9), can force the charge fields to follow the spin fields parasitically. On the ordered side, this implies that the charge order parameter grows quadratically slower than the spin order parameter [14]. Defining $r_{M} \sim(g-$ $\left.g_{\mathrm{c}}\right) / g_{\mathrm{c}}$ ( $g$ is the bare coupling constant and $g_{\mathrm{c}}$ the critical coupling) and $\beta$ as the order parameter exponent of the $Z_{2} \times O(3)$ transition,

$$
\begin{aligned}
& \vec{M} \sim\left(\frac{g_{\mathrm{c}}-g}{g_{\mathrm{c}}}\right)^{\beta}, \\
& N \sim\left(\frac{g_{\mathrm{c}}-g}{g_{\mathrm{c}}}\right)^{2 \beta},
\end{aligned}
$$

a behavior which can easily be checked experimentally by, e.g., measuring the increase of the spin- and charge superlattice peaks as function of increasing $N d$ concentration.

This 'slavery' of the charge field to the spin fields is also expected to hold in the quantum disordered regime close enough to the transition. The arguments is as follows: in the neighborhood of the spin transition, where $r_{M}$ changes sign, the charge sector is still in the disordered regime, implying a charge-correlation length $\xi_{N} \sim 1 / \sqrt{r_{N}}$ or a charge mass gap $\Delta_{N}=$ $c_{N} / \xi_{N} \sim c_{N} \sqrt{r_{N}}$. For lengths $\gg \xi_{N}$ (energies $<\Delta_{N}$ ), these fields can be integrated out by taking their saddlepoint values. Minimizing $S_{\text {sstripes }}$ (Eq. (10)) to the charge fields,

$N_{q}=\frac{\lambda_{1}}{4\left(r_{N} / 2+q^{2}\right)} \sum_{i} M_{i, q}^{2}$

including the gradient terms ( $q$ is Euclidean momentum). After substitution of Eq. (13) in the full action Eq. (10) a spin-only action is obtained with a renormalised quartic term $u_{M} / 4$ ! $\rightarrow u_{M} / 4 !-\lambda_{1}^{2} /\left(2 r_{N}\right)$. As long as this quantity is positive, the critical dynamics is in the spin-only $\left(Z_{2} \times O(3)\right)$ universality class. This implies that the charge-field does not 
carry any dynamics of its own, but follows instead adiabatically the spin dynamics. This has interesting consequences for the charge dynamics. Using Eq. (13) the (dynamical) charge susceptibility becomes in terms of the euclidean momentum $q$,

$$
\begin{aligned}
\chi_{q}^{N} & =\left\langle N_{q} N_{-q}\right\rangle \\
& \sim \frac{\lambda_{1}^{2}}{\left(r_{N}+q^{2}\right)^{2}}\left\langle\left(\sum_{i} M_{q, i}^{2}\right)\left(\sum_{j} M_{-q, j}^{2}\right)\right\rangle .
\end{aligned}
$$

This implies that the stripe-like charge fluctuations will exhibit a dynamics which is quite similar to the spin dynamics. For instance, the charge fluctuations will show a quantum gap in the disordered regime which will be identical to the spin gap in the magnetic sector. On a more detailed level, there will be differences. On the Gaussian level $\chi_{q}^{\mathrm{N}} \sim 1 /\left(r_{N}+\right.$ $\left.q^{2}\right)^{2}\left(\chi_{q}^{M}\right)^{2}$ where $\chi_{q}^{M}=\sum_{i}\left\langle M_{i, q} M_{i,-q}\right\rangle$ (dynamical spin susceptibility). However, in the 3D case this will no longer be true because of the relevancy of the four point vertex.

It is noticed that it remains to be established how the critical fluctuations associated with this transition interact with the 'background' superconductor. In the charged quasi-2D superfluid, the action Eq. (5) describes the acoustic plasmon, keeping in mind that the $c$-axis Josephson plasma frequency sets a low energy cut-off. The arguments by Frey and Balents [13] for the supersolid transition, as discussed in Section 3.2, can now be directly transferred to the case of a pure spin transition (the specific heat exponent $\alpha<0$ for $O(3)$ ). The subtlety is, however, that the spin ordering is accompanied by the breaking of spatial rotational symmetry (the two stripe directions), which changes the universality class of the transition to $Z_{2} \times O(3)$ and this has to be studied in further detail.

Finally, there is a serious problem with this scenario. In the above I asserted that the zero temperature phase diagram has to do with the spin driven transition of Zachar et al. At the same time, in the LTT stripe phases the finite temperature transitions in the stripe ordered region of the $T=0$ phase diagram are of the charge driven kind: charge orders at a higher temperature than the stripe antiferromagnet. At least in the close neighborhood of the quan- tum phase transition, where the GLW theory is valid, such a finite temperature behavior appears as impossible. In strictly $2+1$ dimensions, any finite temperature will destroy the spin order, and it is easy to understand that in the realistic case (spin anisotropy, $3+1$ D couplings) the spin ordering temperature can become quite low due to the fluctuations. The problem is, however, that in the close neighborhood of the quantum transition the charge sector does not show a tendency to order in the absence of the spins. In order to find a finite temperature charge ordering transition, it is necessary to renormalize $r_{N}$ from a large positive value at $T=0$ to a negative value at any finite temperature. Since temperature acts in quantum field theory like a finite size scaling, it is hard to see how this can happen.

\section{Conclusions}

I have presented here a minimal option for the phenomenological theory of the zero temperature competition between superconducting- and stripe order. It is based on current beliefs on the types of order relevant for the cuprates. The identification of these orders is based on a still highly incomplete experimental characterization. At the same time, I hope I have convinced the readership that by elementary considerations a variety of predictions can be derived. It is hoped that these issues are taken up by the experimentalists, who are in the position to prove the above right or wrong.

Let me end this discussion by commenting on some distinct, but closely related ideas: (i) Laughlin argues that the coexistence phase, critical behaviors, etcetera, are not an intrinsic property of the clean limit but instead are caused by dirt effects [47]. As repeatedly emphasized, first-order behavior is rather natural in the present context. Laughlin argues that the 'most relevant operator' quenched disorder changes this into a (pseudo) continuous behavior, while the coexistence phase is a strongly disordered micro-phase-separated affair of insulating stripes and pure superconductors. Although this possibility is not excluded, I repeat that it is not easy to understand how to arrive at the spin quantum criticality claimed by Aeppli et al. (ii) The quantum liquid crystals as 
proposed by Kivelson et al. [27,28]. There is no conflict between those ideas and what is presented here. The liquid crystal ideas amount to the assertion that the charge sector might reveal a substructure which is more complex than the simple density wave order which has been considered here. (iii) The 'unified' $S O(5)$ ideas of Zhang [48]. It is actually the case that the phenomenology presented here can be completely reformulated in terms of a $S O(5)$ action, if appropriate anisotropies are added. For instance, the antiferromagnetic supersolid can be understood as a 'canted' superspin phase, where the $S O(5)$ vector is canted in a direction in between the magnetic and superconducting directions ( $\pi$ mode condensation). A difference with the original $S O(5)$ proposal [48] is that the antiferromagnetic component is now associated with the finite wavevector stripe antiferromagnet, instead of the commensurate magnet of half-filling. Assuming that a mildly broken $S O(5)$ symmetry is governing the dynamics gives rise to a number of additional possibilities. For instance, finite momentum superconductivity appears as a serious possibility within the $S O(5)$ framework: the simplest superconducting stripe phase corresponds with a $S O(5)$ spiral where the superspin rotates from magnetic to superconducting directions. It follows immediately that the superconductivity lives at the same wavevectors as the stripe antiferromagnet. Obviously, the most striking specialty of $S O(5)$ is that the full symmetry can get restored at isolated point(s) in the zero temperature phase diagram, such that superconductivity and antiferromagnetism occur on a strictly equal footing.

\section{Acknowledgements}

I acknowledge stimulating discussions with G.A. Aeppli, V.J. Emery, R.J. Laughlin, S. Sachdev, J.M. Tranquada, A. van Otterlo, W. van Saarloos, and S.-C. Zhang.

\section{References}

[1] J.M. Tranquada, B.J. Sternlieb, J.D. Axe, Y. Nakamura, S. Uchida, Nature 375 (1995) 561, see also J.M. Tranquada, cond-mat/9802034 and refs. therein.
[2] J.M. Tranquada et al., Phys. Rev. Lett. 78 (1997) 338.

[3] S. Sachdev, Quantum Phase Transitions, Cambridge Univ. Press, to be published.

[4] S. Sachdev, in: B.-L. Hao (Ed.), Proc. of the 19th IUPAP International Conference on Statistical Physics, World Scientific, Singapore, 1996; cond-mat/9508080.

[5] G.A. Aeppli et al., Science 278 (1998) 1432.

[6] K. Yamada et al., Phys. Rev. 57 (1998) 6165.

[7] K. Yamada et al., Phys. Rev. Lett. 75 (1995) 1626.

[8] C.N.A. van Duin, J. Zaanen, Phys. Rev. Lett. 80 (1998) 1513.

[9] C. Bruder, R. Fazio, G. Schön, Phys. Rev. B 47 (1993) 342.

[10] G.G. Batrouni, R.T. Scalettar, G.T. Zimanyi, A.P. Kampf, Phys. Rev. Lett. 74 (1995) 2527.

[11] R.T. Scalettar, G.G. Batrouni, A.P. Kampf, G.T. Zimanyi, Phys. Rev. B 51 (1995) 8467

[12] A. van Otterlo et al., Phys. Rev. B 52 (1995) 16176.

[13] E. Frey, L. Balents, Phys. Rev. B 55 (1997) 1050.

[14] O. Zachar, S.A. Kivelson, V.J. Emery, Phys. Rev. B 57 (1998) 1422.

[15] B. Büchner, M. Breuer, A. Freimuth, A.P. Kampf, Phys. Rev. Lett. 73 (1994) 1841.

[16] A.R. Moodenbaugh, L.H. Lewis, S. Soman, Physica C 290 (1997) 98.

[17] K. Hirota, K. Yamada, I. Tanaka, H. Kojima, Physica B, in press.

[18] A.H. Castro-Neto, A.V. Balatski, cond-mat/9805273.

[19] N. Hasselman et al., cond-mat/9807070.

[20] G.S. Boebinger et al., Phys. Rev. Lett. 77 (1996) 5417.

[21] D. Arovas et al., Phys. Rev. Lett. 79 (1997) 2871.

[22] Y. Fukuzumi, K. Mizuhashi, K. Takenaka, S. Uchida, Phys. Rev. Lett. 76 (1996) 684.

[23] Ch. Niedermayer et al., Phys. Rev. Lett. 80 (1998) 3843.

[24] T. Suzuki et al., Phys. Rev. B 57 (1998) R3229.

[25] B.J. Suh et al., Phys. Rev. Lett. (in press, condmat/9804200).

[26] P.C. Hammel et al., cond-mat/9809096.

[27] S.A. Kivelson, E. Fradkin, V.J. Emery, Nature 393 (1998) 550.

[28] S.A. Kivelson, V.J. Emery, cond-mat/9809082.

[29] D. Pines, Z. Phys. B 103 (1997) 129, and refs. therein.

[30] J.W. Loram et al., Physica C 235-240 (1994) 134.

[31] J.W. Loram et al., Physica C 282-287 (1997) 1405.

[32] J.A. Hertz, Phys. Rev. B 14 (1976) 525.

[33] A.J. Millis, Phys. Rev. B 48 (1993) 7183.

[34] C. Castellani, C. Di Castro, M. Grilli, Phys. Rev. Lett. 75 (1995) 4650.

[35] P.W. Anderson, The Theory of Superconductivity in the High $T_{\mathrm{c}}$ Cuprates, Princeton Univ. Press, Princeton, 1997.

[36] A.I. Larkin, Zh. Eksp. Teor. Fiz. 58 (1970) 1466, Sov. Phys. JETP 31 (1970) 784.

[37] T. Dombre, N. Read, Phys. Rev. B 39 (1989) 6797.

[38] J. Zaanen, J. Phys. Chem. Sol. (in press, cond-mat/9711009) and refs. therein.

[39] J.M. Tranquada, J.E. Lorenzo, D.J. Buttrey, V. Sachan, Phys. Rev. B 52 (1995) 3581.

[40] S.R. White, D.J. Scalapino, Phys. Rev. Lett. 80 (1998) 1272. 
[41] O. Nayak, F. Wilczek, Phys. Rev. Lett. 78 (1997) 2465.

[42] J. Zaanen, A.M. Oleś, Ann. Physik 5 (1996) 224.

[43] G. Seibold, C. Castellani, C. Di Castro, M. Grilli, condmat $/ 9803184$.

[44] D.S. Fisher, P.C. Hohenberg, Phys. Rev. B 37 (1988) 4936.

[45] E. Brezin, J.C. Le Guillou, J. Zinn-Justin, in: C. Domb, M.S. Green (Eds.), Phase Transitions and Critical Phenonema, Vol. 6, Academic Press, London, 1976.
[46] J. Tworzydlo, O.Y. Osman, C.N.A. van Duin, J. Zaanen, submitted to Phys. Rev. B (cond-mat/9804012).

[47] R.B. Laughlin, unpublished.

[48] S.-C. Zhang, Science 275 (1997) 1089.

[49] C. Castellani, C. di Castro, M. Grilli, Z. Phys. B 103 (1997) 137.

[50] A. Perali, C. Castellani, C. di Castro, M. Grilli, Phys. Rev. B 54 (1996) 16216. 